\title{
Entorhinal Cortex: Antemortem Cortical Thickness and Postmortem Neurofibrillary Tangles and Amyloid Pathology
}

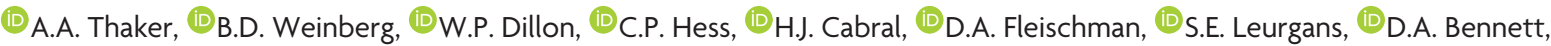

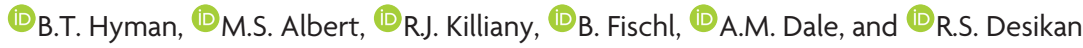

\begin{abstract}
BACKGROUND AND PURPOSE: The entorhinal cortex, a critical gateway between the neocortex and hippocampus, is one of the earliest regions affected by Alzheimer disease-associated neurofibrillary tangle pathology. Although our prior work has automatically delineated an MR imaging-based measure of the entorhinal cortex, whether antemortem entorhinal cortex thickness is associated with postmortem tangle burden within the entorhinal cortex is still unknown. Our objective was to evaluate the relationship between antemortem MRI measures of entorhinal cortex thickness and postmortem neuropathological measures.
\end{abstract}

MATERIALS AND METHODS: We evaluated 50 participants from the Rush Memory and Aging Project with antemortem structural T7-weighted MR imaging and postmortem neuropathologic assessments. Here, we focused on thickness within the entorhinal cortex as anatomically defined by our previously developed MR imaging parcellation system (Desikan-Killiany Atlas in FreeSurfer). Using linear regression, we evaluated the association between entorhinal cortex thickness and tangles and amyloid- $\beta$ load within the entorhinal cortex and medial temporal and neocortical regions.

RESULTS: We found a significant relationship between antemortem entorhinal cortex thickness and entorhinal cortex $(P=.006)$ and medial temporal lobe tangles $(P=.002)$; we found no relationship between entorhinal cortex thickness and entorhinal cortex $(P=.09)$ and medial temporal lobe amyloid- $\beta(P=.09)$. We also found a significant association between entorhinal cortex thickness and cortical tangles $(P=.003)$ and amyloid- $\beta(P=.01)$. We found no relationship between parahippocampal gyrus thickness and entorhinal cortex $(P=.31)$ and medial temporal lobe tangles $(P=.051)$.

CONCLUSIONS: Our findings indicate that entorhinal cortex-associated in vivo cortical thinning may represent a marker of postmortem medial temporal and neocortical Alzheimer disease pathology.

ABBREVIATIONS: $\mathrm{AD}=$ Alzheimer disease; $\mathrm{EC}=$ entorhinal cortex; $\mathrm{SE}=$ standard error

$\mathbf{T}$ he human entorhinal cortex (EC) plays an integral role in memory formation and serves as the critical gateway between

the hippocampus and neocortex. ${ }^{1}$ Located in the medial temporal lobe, the EC constitutes the anterior portion of the parahip-

Received June 13, 2016; accepted after revision January 10, 2017

From the Department of Radiology (A.A.T.), University of Colorado School of Medicine, Aurora, Colorado; Department of Radiology and Imaging Sciences (B.D.W.), Emory University Hospital, Atlanta, Georgia; Neuroradiology Section (W.P.D., C.P.H., R.S.D.), Department of Radiology and Biomedical Imaging, University of California, San Francisco, San Francisco, California; Departments of Biostatistics (H.J.C.) and Anatomy and Neurobiology (R.J.K.), Boston University School of Public Health, Boston, Massachusetts; Rush Alzheimer's Disease Center (D.A.F., S.E.L., D.A.B.), Rush University Medical Center, Chicago, Illinois; Department of Neurology (B.T.H.), Massachusetts General Hospital, Boston, Massachusetts; Department of Neurology and Division of Cognitive Neurosciences (M.S.A.), Johns Hopkins University, Baltimore, Maryland; Athinoula A. Martinos Center for Biomedical Imaging (B.F.), Department of Radiology, Massachusetts General Hospital, Charlestown, Massachusetts; Computer Science and Artificial Intelligence Laboratory (B.F.), Massachusetts Institute of Technology, Cambridge, Massachusetts; and Departments of Radiology (A.M.D.), Cognitive Sciences and Neurosciences, University of California, San Diego, La Jolla, California.

This work was supported by National Institutes of Health grants R01AG10161 and R01AG40039. R.S.D. was supported by the National Alzheimer's Coordinating Center Junior Investigator Award, ASNR Foundation Alzheimer's Imaging Research Grant Program, RSNA Resident/Scholar Award, and a National Institutes of
Health/National Institute on Drug Abuse grant U24DA041123. Support for this research was provided in part by the National Institute for Biomedical Imaging and Bioengineering (P41EB015896, R01EB006758, R21EB018907, R01EB019956), the National Institute on Aging (5R01AG008122, R01AG016495), and the National Institute for Neurological Disorders and Stroke (R01NS0525851, R21NS072652, R01NS070963, R01NS083534, 5U01NS086625) and was made possible by the resources provided by Shared Instrumentation Grants 1S10RR023401, 1S10RR019307, and 1S10RR023043. Additional support was provided by the National Institutes of Health Blueprint for Neuroscience Research (5U01-MH093765), part of the multi-institutional Human Connectome Project. In addition, B.F. has a financial interest in CorticoMetrics, a company whose medical pursuits focus on brain imaging and measurement technologies. B.F.'s interests were reviewed and are managed by Massachusetts General Hospital and Partners HealthCare in accordance with their conflict of interest policies. A.M.D. is a founder and holds equity in CorTechs Labs Inc and also serves on the Scientific Advisory Board of CorTechs Labs and Human Longevity Inc. The terms of these arrangements have been reviewed and approved by the University of California, San Diego, in accordance with its conflict of interest policies.

Please address correspondence to Rahul S. Desikan, MD, PhD, Department of Radiology and Biomedical Imaging, University of California, San Francisco, 505 Parnassus Ave San Francisco, CA 94143; e-mail: rahul.desikan@ucsf.edu

- Indicates open access to non-subscribers at www.ajnr.org

http://dx.doi.org/10.3174/ajnr.A5133 
pocampal gyrus and is localized in vivo laterally by the rhinal sulcus, anteriorly by the amygdala and hippocampus, and posteriorly by the posterior portion of the parahippocampal gyrus. ${ }^{2,3}$ The EC is one of the earliest affected regions in Alzheimer disease (AD). Tau-associated neurofibrillary tangle pathology in AD follows a defined topographic and hierarchical pattern, first affecting the EC and then progressing to anatomically connected limbic and association cortices; in contrast, amyloid- $\beta$-associated pathology does not involve the $\mathrm{EC}$ in the earliest stages of $\mathrm{AD}$ but selectively affects the neocortical regions. ${ }^{4,5}$ Most important, the association of neuronal volume loss in the EC has been shown to parallel $\tau$-associated tangle pathology (neurofibrillary tangles and neuritic plaques) but not senile plaques seen with amyloid- $\beta$ deposition. ${ }^{4}$

Structural MR imaging provides visualization and quantification of volume loss and has been extensively investigated in $\mathrm{AD}$. $^{6-9}$ Using manually delineated assessments, early studies have shown that volumetric measures of the EC can identify individuals without dementia in the earliest stages of the AD process. ${ }^{7-9}$ Within the past decade, rapid advances in MR imaging postprocessing have led to the development of software tools for automatic quantification of human subcortical and neocortical regions. ${ }^{10} \mathrm{We}$ have previously developed an MR imaging-based parcellation atlas for the human cerebral cortex, which has automatically delineated the entorhinal cortex. ${ }^{11}$ The EC ROI from our parcellation atlas correlates with CSF levels of $\tau$, amyloid, ${ }^{12}$ and Apolipoprotein $E^{13}$ and has been used to identify cognitively healthy ${ }^{14}$ and cognitively impaired individuals without dementia who are most likely to progress to clinical AD. ${ }^{15,16}$ However, whether our antemortem MR imaging-based measure of the EC is associated with established postmortem measures of $\mathrm{AD}$ pathology is still unknown.

In this study, we evaluated the relationship between antemortem MR imaging-based automated measurements of EC thickness and postmortem measures of neurofibrillary tangle and amyloid- $\beta$ pathology. To assess the specificity of our EC ROI (anterior portion of the parahippocampal gyrus), we also evaluated the relationship between the thickness of the posterior parahippocampal gyrus and EC amyloid- $\beta$ and $\tau$ pathology.

\section{MATERIALS AND METHODS \\ Participants}

We evaluated participants from the Rush Memory and Aging Project, a community-based longitudinal study of aging, which began in 1997. ${ }^{17}$ Details of the clinical and neuropathologic evaluations have been reported previously. ${ }^{17,18}$ Briefly, all participants underwent a uniform structured clinical evaluation that included a medical history, physical examination with emphasis on neurologic function, and neuropsychological testing (including the Mini-Mental State Examination and 20 other tests). All participants were evaluated in person by a neuropsychologist and a physician with expertise in the evaluation of older individuals with cognitive impairment. On the basis of physician evaluation and review of the cognitive testing and the neuropsychologist's opinion, participants were classified with respect to $\mathrm{AD}$ and other common conditions with the

\begin{tabular}{|c|c|c|c|}
\hline & $\begin{array}{l}\text { Healthy } \\
(n=25)\end{array}$ & $\begin{array}{c}\mathrm{MCl} \\
(n=18)\end{array}$ & $\begin{array}{c}\text { AD } \\
(n=7)\end{array}$ \\
\hline$\%$ Female & $64 \%$ & $55 \%$ & $71 \%$ \\
\hline Education (yr) & $15.6(3.6)$ & $14.9(1.2)$ & $13.2(1.2)$ \\
\hline Age at MRI (yr) & $87.4(5.0)$ & $88.3(5.4)$ & $85.2(4.4)$ \\
\hline Age at death (yr) & $90.3(4.7)$ & $91.1(5.4)$ & $88.7(4.9)$ \\
\hline Years between MRI and death & $2.8(1.3)$ & $2.8(1.2)$ & $3.6(1.5)$ \\
\hline Entorhinal cortex thickness & $1.50(0.30)$ & $1.49(0.27)$ & $1.40(0.41)$ \\
\hline EC tangle density & $8.9(6.2)$ & $12.8(10.3)$ & $20.1(12.9)$ \\
\hline EC amyloid- $\beta$ load & $5.5(5.1)$ & $7.1(5.1)$ & $7.5(4.9)$ \\
\hline
\end{tabular}

Note:- $\mathrm{MCl}$ indicates mild cognitive impairment.

${ }^{a}$ All values are expressed as mean (SD).

potential to impact cognitive function according to the recommendations of the joint working group of the National Institute of Neurologic and Communicative Disorders and Stroke and the Alzheimer's Disease and Related Disorders Association. ${ }^{19}$ In this article, we focused on 50 participants, clinically defined at baseline (on study entry) as being cognitively healthy $(n=25)$, having mild cognitive impairment $(n=18)$, and having probable $\mathrm{AD}(n=7)$ (Table), with concurrent antemortem MR imaging and postmortem neuropathologic assessments. The Rush University Medical Center institutional review board approved the study, and all participants gave written informed consent and signed an Anatomic Gift.

\section{Imaging Assessments}

We assessed previously obtained T1-weighted anatomic data by using a $1.5 \mathrm{~T}$ MR imaging scanner (GE Healthcare, Milwaukee, Wisconsin). For the current study, all antemortem MR imaging data were acquired by using a 3D magnetization-prepared rapid acquisition of gradient echo sequence with the following parameters: $\mathrm{TE}=2.8 \mathrm{~ms}, \mathrm{TR}=6.3 \mathrm{~ms}$, preparation time $=1000 \mathrm{~ms}$, flip angle $=8^{\circ}$, FOV $=24 \times 24 \mathrm{~cm}, 160$ sections, $1-\mathrm{mm}$ section thickness, a $224 \times 192$ acquisition matrix reconstructed to $256 \times$ 256 , and 2 repetitions. The MR imaging data were automatically segmented with FreeSurfer 5.0 (http://surfer.nmr.mgh.harvard. edu; for additional details see McKhann et $\mathrm{al}^{19}$ ). Here, we focused on intracranial volume-corrected average thickness of the entire entorhinal cortex and posterior parahippocampal gyrus (average of the left and right hemispheres) as delineated with our previously developed automated cortical parcellation atlas (DesikanKilliany atlas in Freesurfer) (Fig 1). ${ }^{11}$ In secondary analyses, we also evaluated baseline intracranial volume-corrected hippocampal volumes (average of the left and right hemispheres). ${ }^{20}$

\section{Neuropathologic Assessments}

We used results from previously obtained neuropathologic evaluations and focused on amyloid and neurofibrillary tangle pathology within the entorhinal cortex and medial temporal (entorhinal and hippocampal) regions (for additional details on neuropathologic measures from the Rush Memory and Aging Project/Rush University Medical Center, please see Bennett et $\mathrm{al}^{21,22}$ and Barnes et $\mathrm{al}^{23}$ ). Briefly, at least 2 tissue blocks from the entorhinal cortex and hippocampus (CA1/subiculum) were dissected from $1-\mathrm{cm}$ coronal slabs fixed for $48-72$ hours in $4 \%$ paraformaldehyde, embedded in paraffin, and cut into $20-\mu \mathrm{m}$ sections. Amyloid- $\beta$ was labeled with MO0872 (1:100; Dako, 


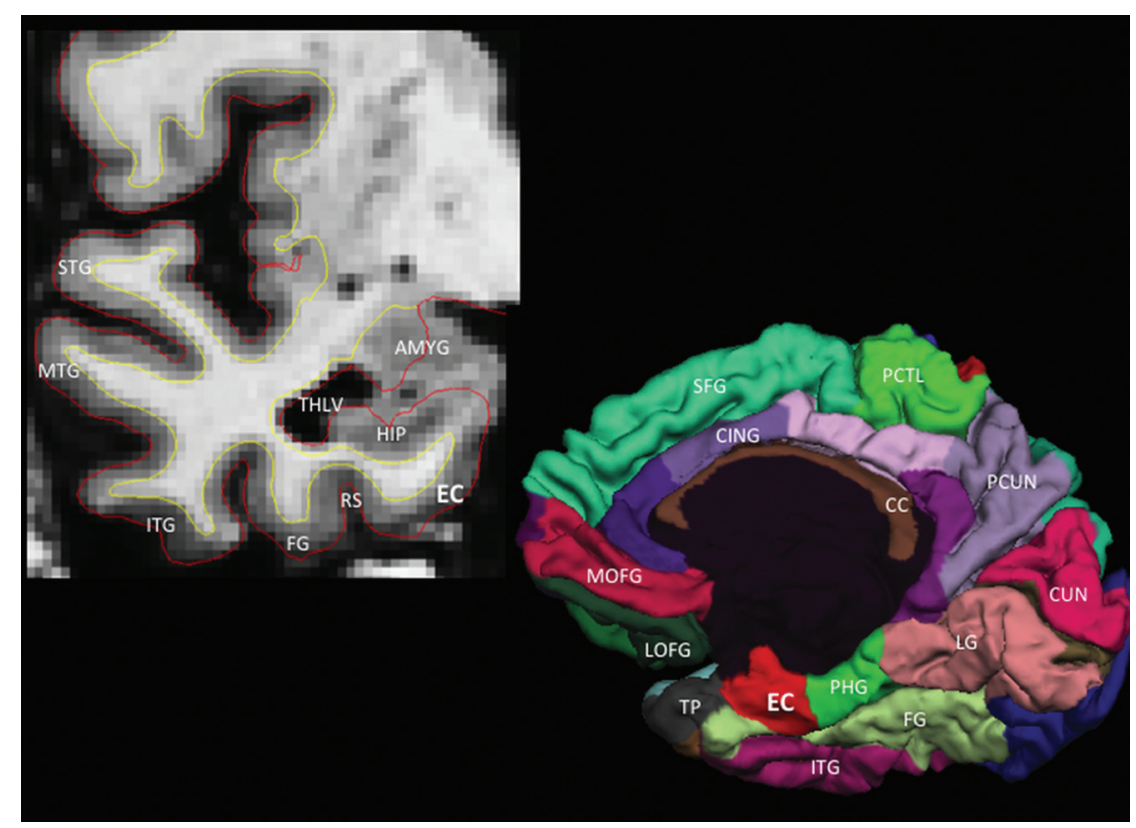

FIG 1. Coronal T1-weighted MR imaging illustrating the anatomic location of the entorhinal cortex, which is medial to the rhinal sulcus (RS) and fusiform gyrus (FG) and inferior to the hippocampus (HIP), temporal horn of the lateral ventricle (THLV), and amygdala (AMYG) (upper left panel). 3D cortical (pial) representation is of the right cortical (pial) surface, delineating the location of the entorhinal cortex on the medial hemisphere of the cerebral cortex (lower right panel). STG indicates superior temporal gyrus; MTG, middle temporal gyrus; ITG, inferior temporal gyrus; TP, temporal pole; PHG, parahippocampal gyrus; LG, lingual gyrus; CUN, cuneus cortex; PCUN, precuneus; FG, fusiform gyrus; PCTL, paracentral lobule; SFG, superior frontal gyrus; MOFG, medial orbitofrontal gyrus; LOFG, lateral orbitofrontal gyrus; CC, corpus callosum; CING, cingulate cortex.
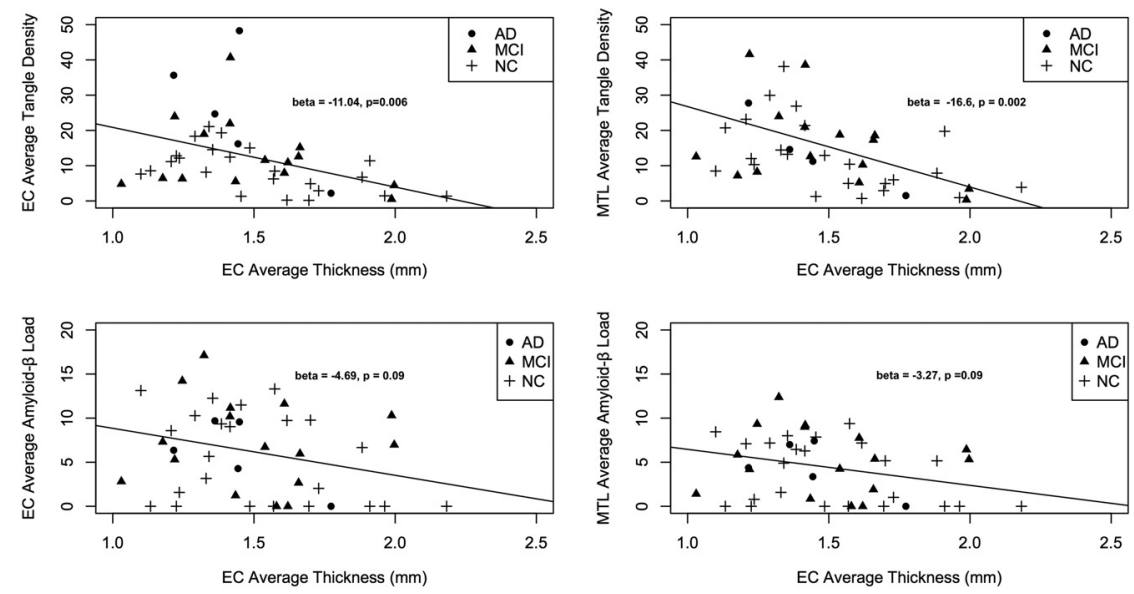

FIG 2. Scatterplots illustrating the relationship between average entorhinal cortex thickness and average tangle density within the entorhinal cortex (upper left) and medial temporal lobe (MTL, upper right), and average amyloid- $\beta$ load within the EC (lower left) and medial temporal lobe (lower right). Best-fit regression line, $\beta$-coefficients, and $P$ values from the logistic regression model are included (for additional details see the text).

Carpinteria, California), and paired helical filament $\tau$ was labeled with AT8 (1:800 in 4\% horse serum; Innogenex, San Ramon, California), an antibody specific for phosphorylated $\tau$. Images of amyloid- $\beta$-stained sections were captured for quantitative analysis by using a systematic random-sampling scheme, and calculation of the percentage area occupied by amyloid- $\beta$ immunoreactive pixels was performed. Quantification of tangle density per square millimeter was performed with a stereologic mapping station. We used composite summary measures of the percentage area occu- pied by amyloid- $\beta$ and the density of neurofibrillary tangles by averaging the values for each lesion within the entorhinal cortex and medial temporal regions (entorhinal cortex and hippocampus) (for additional details see Bennett et $\mathrm{al}^{21,22}$ and Barnes et $\mathrm{al}^{23}$ ). To evaluate neuropathology within the neocortex and to minimize multiple comparisons, we used a composite measure of tangle density and amyloid- $\beta$ load within the midfrontal cortex, inferior temporal gyrus, inferior parietal cortex, calcarine cortex, cingulate region, and superior frontal gyrus (cortical tangle density and cortical amyloid- $\beta$ load).

\section{Statistical Analysis}

Using linear regression, we evaluated the association between entorhinal cortex thickness and average tangle and amyloid- $\beta$ load within the entorhinal cortex and medial temporal regions (entorhinal cortex + hippocampus). We also evaluated the relationship between entorhinal cortex thickness and cortical tangle density and amyloid- $\beta$ load. In secondary analyses, we assessed the association between (posterior) parahippocampal gyrus thickness and tangles and amyloid- $\beta$ load within the entorhinal cortex and medial temporal regions. In all analyses, we controlled for the effects of age at death, sex, and clinical diagnosis.

\section{RESULTS}

We found a relationship between antemortem entorhinal cortex thickness and postmortem tangle density within the entorhinal cortex $(\beta$-coefficient $=$ -11.04 , standard error $[\mathrm{SE}]=3.78, P$ value $=.006)$ and medial temporal regions $(\beta$-coefficient $=-16.67, \mathrm{SE}=$ $5.04, P=.002)$; lower EC thickness was associated with increased EC and medial temporal lobe tangle density (Fig 2). Even after controlling for the effects of hippocampal volume, the relationship between entorhinal cortex thickness and tangle density within the entorhinal cortex $(\beta$-coefficient $=-9.36, \mathrm{SE}=2.57, P=.03)$ and medial temporal regions $(\beta$-coefficient $=-12.89, \mathrm{SE}=5.64, P=$ $.02)$ remained significant. We found no relationship between entorhinal cortex thickness and amyloid- $\beta$ load within the entorhinal $\operatorname{cortex}(\beta$-coefficient $=-4.69, \mathrm{SE}=2.70, P=.09)$ and medial temporal regions $(\beta$-coefficient $=-3.27, \mathrm{SE}=1.90, P=.09)($ Fig 2$)$.

We found a relationship between entorhinal cortex thickness and both cortical tangle density $(\beta$-coefficient $=-37.5, \mathrm{SE}=$ 

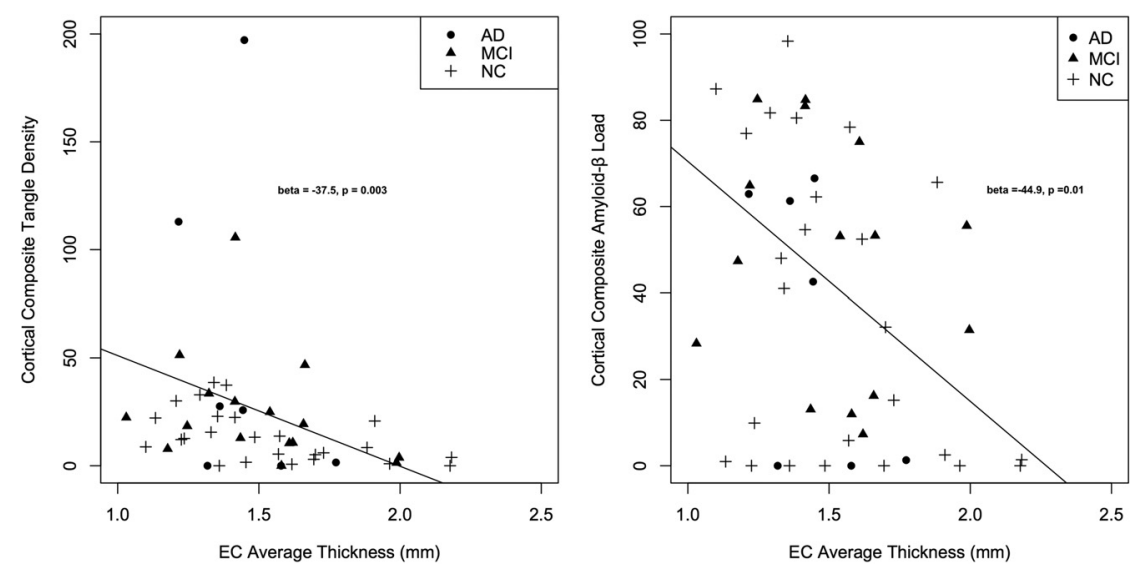

FIG 3. Scatterplots illustrating the relationship between average entorhinal cortex thickness and composite tangle density (left) and amyloid- $\beta$ load (right) within the cerebral cortex (see text for details). Best-fit regression line, $\beta$-coefficients, and $P$ values from the logistic regression model are included (for additional details see the text).

12.2, $P=.0003)$ and amyloid- $\beta$ load $(\beta$-coefficient $=-44.9$, $\mathrm{SE}=17.2, P=.01)($ Fig 3$)$. Even after we controlled for the effects of hippocampal volume, the relationship between entorhinal cortex and both cortical tangle density $(\beta$-coefficient $=-34.4, \mathrm{SE}=$ $7.9, P=.01)$ and amyloid- $\beta$ load $(\beta$-coefficient $=-44.7, \mathrm{SE}=$ $18.6, P=.02)$ remained significant.

In contrast, we found no relationship between antemortem parahippocampal gyrus thickness and postmortem tangle density within the entorhinal cortex $(\beta$-coefficient $=-4.04, \mathrm{SE}=4.33$, $P=.31)$ and a trend toward significance for the medial temporal regions $(\beta$-coefficient $=-11.52, \mathrm{SE}=5.71, P=.051)$. Similarly, we found no relationship between parahippocampal gyrus thickness and amyloid load within the entorhinal cortex $(\beta$-coefficient $=$ $1.02, \mathrm{SE}=2.71, P=.709)$ and medial temporal regions $(\beta$-coefficient $=0.57, \mathrm{SE}=1.91, P=.766)$. We also found no relationship between parahippocampal gyrus thickness and cortical tangle density $(\beta$-coefficient $=-14.2, \mathrm{SE}=13.5, P=.30)$ and amyloid- $\beta$ load $(\beta$-coefficient $=-8.7, \mathrm{SE}=18.6, P=.64)$.

We performed subgroup analyses within our subset of cognitively healthy older participants $(n=25)$ to evaluate the relationship between MR imaging measures of entorhinal cortex thickness and neuropathology. Similar to our main results, we found a relationship between antemortem entorhinal cortex thickness and postmortem tangle density within the entorhinal cortex $(\beta$ coefficient $=-10.32, \mathrm{SE}=4.03, P=.01)$, medial temporal regions $(\beta$-coefficient $=-14.48, \mathrm{SE}=6.61, P=.04)$, and cortex $(\beta$-coefficient $=-16.58, \mathrm{SE}=7.03, P=.02)$. In contrast, we found no relationship between entorhinal cortex thickness and amyloid load either within the entorhinal cortex $(\beta$-coefficient $=$ $-5.04, \mathrm{SE}=3.91, P=.23)$ or cortex $(\beta$-coefficient $=-32.26$, $\mathrm{SE}=22.79, P=.12$ ).

\section{DISCUSSION}

Our results demonstrate that quantitative in vivo volumetric MR imaging measurements of the EC are associated with postmortem measures of entorhinal and neocortical AD pathology. Specifically, these results indicate that lower EC thickness predicts higher postmortem tangle load. We also found a similar association between EC thickness and postmortem tangle load within the medial temporal regions (EC + hippocampus). Most important, this effect remained significant after controlling for hippocampal volume. Finally, we found a robust relationship between EC thickness and cortical tangle and amyloid- $\beta$ pathology. Rather than representing a specific measure of EC tangle pathology, our combined findings suggest that an antemortem MR imaging measure of the entorhinal cortex likely captures Alzheimer-associated pathology within the medial temporal and neocortical regions.

Using neuropathologic assessments from the same individuals and building on our prior work, ${ }^{12,14}$ this study suggests that EC-associated cortical thinning in $\mathrm{AD}$ may represent a marker of $\tau$-associated pathology within both the entorhinal cortex and neocortex. Consistent with the known pattern of amyloid- $\beta$ deposition in $\mathrm{AD}$, which heavily involves the neocortex with relative sparing of the entorhinal cortex, an association was found between EC thickness and neocortical amyloid- $\beta$ load but not entorhinal amyloid pathology, compatible with the hypothesis that amyloid deposition within the neocortex, rather than the EC may represent an early component of Alzheimer pathobiology. ${ }^{24,25}$ Our results also suggest that entorhinal cortex thickness may provide independent information about $\mathrm{AD}$ pathology even after accounting for hippocampal volume, further illustrating the importance of evaluating EC thickness as an early marker of in vivo Alzheimer neurodegeneration. Most interesting, even among cognitively healthy older adults, we found a selective relationship between entorhinal cortex thickness and tangle pathology but not amyloid pathology, suggesting the potential usefulness of quantitative MR imaging measures in preclinical AD.

Accumulating evidence suggests that $\tau$ pathology is closely associated with cognitive performance, particularly in the early stages of disease. ${ }^{26,27}$ These findings suggest that automated measures of entorhinal cortex atrophy may reflect regional $\tau$ pathology, which will be clinically useful for early $\mathrm{AD}$ detection and disease monitoring. Additionally, quantitative measures of EC and medial temporal structures may be combined with genetic, fluid (CSF or plasma), and cognitive parameters for risk stratification, which may become increasingly relevant for AD prevention and therapeutic trials. With the advent and use of novel agents for detecting in vivo $\tau$ deposition, ${ }^{28}$ volumetric MR imaging could be integrated with PET imaging to determine whether regional measures of entorhinal atrophy and $\tau$ deposition provide independent or complementary information. Finally, beyond $\mathrm{AD}$, automated assessments of the entorhinal cortex can be useful in other disorders of the medial temporal lobe such as medial temporal sclerosis, vascular dementia, and frontotemporal lobar degeneration.

A potential limitation of our study is that our imaging and neuropathology datasets were not coregistered; thus, this drawback limits the precise correspondence between antemortem and 
postmortem definitions of the EC. Another limitation is the need for validation of our results in independent, community-based samples.

\section{CONCLUSIONS}

We found a strong association between an automated, antemortem MR imaging-based measure of EC thickness and postmortem neurofibrillary tangle burden within the entorhinal cortex, medial temporal lobe, and neocortex. We additionally detected a relationship between EC thickness and neocortical amyloid- $\beta$ load. Considered together, our findings serve as a validation of our automated MR imaging measure of the EC and suggest that EC-associated cortical thinning in AD may represent a marker of medial temporal and neocortical AD neuropathology.

\section{ACKNOWLEDGMENTS}

We thank the participants in the Rush Memory and Aging Project.

Disclosures: Sue E. Leurgans-RELATED: Grant: National Institutes of Health, Comments: P30AG010161 R01 NS078009, P20MD006886 P20MD006886-S1 RF1AG015819 U01AG046152-S1 R01AG034374 R01AG047976 R01AG033570.* David A. BennettRELATED: Grant: National Institutes of Health*. Bradley T. Hyman—RELATED: Grant: National Institutes of Health*; UNRELATED: Board Membership: NeuroPhage Pharmaceuticals; Consultancy: Genentech, Eli Lilly and Company, AbbVie, Merck, Biogen; Grants/Grants Pending: Merck, Denali, Spark, AbbVie, Biogen, Intellect*; Stock/ Stock Options: Novartis, Marilyn S. Albert-UNRELATED: Consultancy: Eli Lilly and Company, Dana Foundation Scientific Advisory Board, Comments: member of the Eli Lilly and Company Advisory Board on preclinical Alzheimer disease, member of the Dana Foundation Scientific Advisory Board; Grants/Grants Pending: National Institutes of Health, Alzheimer's Association, Fidelity Biosciences, Bruce Fischl—RELATED: Grant: National Institutes of Health*; UNRELATED: Grants/Grants Pending: National Institutes of Health*; Stock/Stock Options: CorticoMetrics. Anders M. DaleRELATED: Grant: National Institutes of Health, Comments: numerous research grants*; UNRELATED: Board Membership: CorTechs Labs Inc, Comments: I am a founder of and equity holder in CorTechs Labs Inc. I also serve on its scientific advisory board; Consultancy: Human Longevity Inc, Comments: I am a member of the Scientific Advisory Board of Human Longevity Inc; Payment for Lectures Including Service on Speakers Bureaus: GE Healthcare, Comments: I am the Principal Investigator of a comprehensive research agreement between the University of California, San Diego, and GE Healthcare. *Money paid to the institution.

\section{REFERENCES}

1. Squire LR, Zola-Morgan S. The medial temporal lobe memory system. Science 1991;253:1380 - 86 CrossRef Medline

2. Braak H, Braak E. Neuropathological stageing of Alzheimer-related changes. Acta Neuropathol 1991;82:239-59 CrossRef Medline

3. Braak H, Braak E. Staging of Alzheimer's disease-related neurofibrillary changes. Neurobiol Aging 1995;16:271-78; discussion 278-84 CrossRef Medline

4. Gómez-Isla T, Price JL, McKeel DW, et al. Profound loss of layer II entorhinal cortex neurons occurs in very mild Alzheimer's disease. J Neurosci 1996;16:4491-500 Medline

5. Arnold SE, Hyman BT, Flory J, et al. The topographical and neuroanatomical distribution of neurofibrillary tangles and neuritic plaques in the cerebral cortex of patients with Alzheimer's disease. Cereb Cortex 1991;1:103-16 CrossRef Medline

6. Desikan RS, Rafii MS, Brewer JB, et al. An expanded role for neuroimaging in the evaluation of memory impairment. AJNR Am J Neuroradiol 2013;34:2075-82 CrossRef Medline

7. Killiany RJ, Gomez-Isla T, Moss M, et al. Use of structural magnetic resonance imaging to predict who will get Alzheimer's disease. Ann Neurol 2000;47:430-39 Medline

8. Dickerson BC, Goncharova I, Sullivan MP, et al. MRI-derived entorhinal and hippocampal atrophy in incipient and very mild Alzheimer's disease. Neurobiol Aging 2001;22:747-54 CrossRef Medline
9. Killiany RJ, Hyman BT, Gomez-Isla T, et al. MRI measures of entorhinal cortex vs hippocampus in preclinical AD. Neurology 2002;58: 1188-96 CrossRef Medline

10. Fischl B. FreeSurfer. Neuroimage 2012;62:774-81 CrossRef Medline

11. Desikan RS, Ségonne F, Fischl B, et al. An automated labeling system for subdividing the human cerebral cortex on MRI scans into gyral based regions of interest. Neuroimage 2006;31:968-80 CrossRef Medline

12. Desikan RS, Cabral HJ, Hess CP, et al; Alzheimer's Disease Neuroimaging Initiative. Automated MRI measures identify individuals with mild cognitive impairment and Alzheimer's disease. Brain 2009; 132:2048-57 CrossRef Medline

13. Toledo JB, Da X, Weiner MW. CSF Apo-E levels associate with cognitive decline and MRI changes. Acta Neuropathol 2014;127:621-32 CrossRef Medline

14. Desikan RS, McEvoy LK, Thompson WK, et al; Alzheimer's Disease Neuroimaging Initiative. Amyloid- $\boldsymbol{\beta}$ associated volume loss occurs only in the presence of phospho-tau. Ann Neurol 2011;70:657-61 CrossRef Medline

15. Desikan RS, Fischl B, Cabral HJ, et al. MRI measures of temporoparietal regions show differential rates of atrophy during prodromal AD. Neurology 2008;71:819-25 CrossRef Medline

16. Desikan RS, Cabral HJ, Fischl B, et al. Temporoparietal MR imaging measures of atrophy in subjects with mild cognitive impairment that predict subsequent diagnosis of Alzheimer disease. AJNR AmJ Neuroradiol 2009;30:532-38 CrossRef Medline

17. Bennett DA, Schneider JA, Buchman AS, et al. The Rush Memory and Aging Project: study design and baseline characteristics of the study cohort. Neuroepidemiology 2005;25:163-75 CrossRef Medline

18. Schneider JA, Arvanitakis Z, Bang W, et al. Mixed brain pathologies account for most dementia cases in community-dwelling older persons. Neurology 2007;69:2197-204 CrossRef Medline

19. McKhann G, Drachmann D, Folstein M, et al. Clinical diagnosis of Alzheimer's disease: report of the NINCDS-ADRDA Work Group under the auspices of Department of Health and Human Services Task Force on Alzheimer's Disease. Neurology 1984;34:939-44 CrossRef Medline

20. Fischl B, Salat DH, Busa E, et al. Whole brain segmentation: automated labeling of neuroanatomical structures in the human brain. Neuron 2002;33:341-55 CrossRef Medline

21. Bennett DA, Schneider JA, Wilson RS, et al. Education modifies the association of amyloid but not tangles with cognitive function. Neurology 2005;65:953-55 CrossRef Medline

22. Bennett DA, Wilson RS, Arvanitakis Z, et al. Selected findings from the Religious Orders Study and Rush Memory and Aging Project. J Alzheimers Dis 2013;33(suppl 1):S397-403 CrossRef Medline

23. Barnes LL, Schneider JA, Boyle PA, et al. Memory complaints are related to Alzheimer disease pathology in older persons. Neurology 2006;67:1581-85 CrossRef Medline

24. Buckner RL, Snyder AZ, Shannon BJ, et al. Molecular, structural, and functional characterization of Alzheimer's disease: evidence for a relationship between default activity, amyloid, and memory. J Neurosci 2005;25:7709-17 CrossRef Medline

25. Jack CR Jr, Barrio JR, Kepe V. Cerebral amyloid PET imaging in Alzheimer's disease. Acta Neuropathol 2013;126:643-57 CrossRef Medline

26. Brier MR, Gordon $B$, Friedrichsen $\mathrm{K}$, et al. Tau and $\mathrm{A} \boldsymbol{\beta}$ imaging, CSF measures, and cognition in Alzheimer's disease. Sci Transl Med 2016;8:338ra66 CrossRef Medline

27. Soldan A, Pettigrew C, Cai Q, et al. Hypothetical preclinical Alzheimer disease groups and longitudinal cognitive change. JAMA Neurol 2016;73:698-705 CrossRef Medline

28. Holtzman DM, Carrillo MC, Hendrix JA, et al. Tau: from research to clinical development. Alzheimers Dement 2016;12:1033-39 CrossRef Medline 